\title{
Maternal Preeclampsia Predicts Elevated Blood Pressure in 12-Year-Old Children: Evaluation by Ambulatory Blood Pressure Monitoring
}

\author{
SIRPA TENHOLA, EERO RAHIALA, PIRJO HALONEN, ESKO VANNINEN, AND RAIMO VOUTILAINEN \\ Department of Pediatrics [S.T., E.R., R.V.] and Clinical Physiology and Nuclear Medicine [E.V.], and IT Service Centre [P.H.], \\ Kuopio University and University Hospital, FI-70211 Kuopio, Finland
}

\begin{abstract}
Ambulatory blood pressure (ABP) monitoring offers a reliable method for determining blood pressure (BP) in children. The aim of this cohort study was to examine whether maternal preeclampsia is associated with elevated BP in an offspring. The study population consisted of 57 children born to preeclamptic mothers (PRE) and their 57 age- and sex-matched control subjects born to normotensive mothers (non-PRE). We examined the 24-h ABP at $12 \mathrm{y}$ of age in the PRE and non-PRE children. Within the two groups, the association of anthropometric measures, plasma catecholamine (epinephrine $[\mathrm{E}]$, norepinephrine $[\mathrm{NE}]$ ) concentrations, and $\mathrm{ABP}$ was examined. The PRE children had significantly higher mean 24-h systolic and diastolic ABPs than the non-PRE children. The same was true for the mean daytime and nighttime systolic and diastolic ABPs. The PRE boys had higher 24-h systolic ABP than the PRE girls. In the PRE children, high plasma $\mathrm{E}$ concentration and being born small for gestational age (SGA) predicted high systolic 24-h $\mathrm{ABP}$ in logistic regression analysis. In the non-PRE children, high current body mass index (BMI) and high plasma E concentration was associated with high systolic 24-h ABP. In conclusion, systolic and diastolic ABP values were elevated in the PRE children. High plasma E concentration and being born SGA were associated with high systolic 24-h ABP in the PRE children. Presumably maternal preeclampsia affects offspring via several mechanisms, including genetic ones and metabolic consequences of restricted intrauterine growth. (Pediatr Res 59: 320-324, 2006)
\end{abstract}

$\mathrm{P}$ reeclampsia is a pregnancy-specific syndrome with elevated $\mathrm{BP}$ and proteinuria after $20 \mathrm{wk}$ of gestation, occurring in about $5 \%$ to $7 \%$ of all pregnancies. A genetic contribution in preeclampsia has been well described, but the etiology is still largely unknown $(1,2)$. Changes in endothelial function and vasoactive hormones have been proposed as possible pathogenetic mechanisms $(3,4)$. Recently, it was reported that women with a history of preeclampsia exhibit impaired endothelial function up to $1 \mathrm{y}$ after delivery (5).

Women with a prior PRE pregnancy are in increased risk of cardiovascular diseases (6-9). Preeclampsia was found to be

Received June 14, 2005; accepted September 21, 2005

Correspondence: Raimo Voutilainen, M.D., Ph.D., Department of Pediatrics, Kuopio University Hospital, P.O. Box 1777, FI-70211 Kuopio, Finland; e-mail: raimo.voutilainen@uku.fi

S.T. and E.R. contributed equally to this paper.

This study was supported by Kuopio University Hospital, Academy of Finland, Sigrid Jusélius Foundation, Finnish Cultural Foundation, Pediatric Research Foundation, and Maud Kuistila Foundation.

DOI: 10.1203/01.pdr.0000196734.54473.e3 an independent risk factor for angiographically documented coronary artery disease in female subjects younger than $66 \mathrm{y}$ of age (9). Laivuori et al. (10) and Kaaja et al. (11) with their coworkers have shown that PRE women are insulin resistant during and after pregnancy, and hyperinsulinemia may persist even 17 y after a PRE pregnancy. Furthermore, PRE women may also have dyslipidemia, hypertension, and higher BMI in the nonpregnant state than women with normal pregnancies $(11,12)$. Thus, metabolic changes seen in preeclampsia, such as insulin resistance, hypertriglyceridemia, and hypertension, are similar to those in metabolic syndrome $(11,12)$. Consequently, a predisposition to the metabolic syndrome may induce women to develop preeclampsia (12).

Maternal preeclampsia has also been associated with elevated BP in offspring already in childhood and adolescence $(13,14)$. We reported previously that 12 -y-old children born to PRE mothers had higher casual systolic and diastolic BP values than their matched control children born to non-PRE mothers (15). The aim of this study was to examine whether this BP increase holds true throughout the day or represents "white coat hypertension" associating with increased catecholamine secretion during casual blood pressure measurements. For this purpose, we measured 24-h ABP values by an oscillometric ABP device in a well-characterized study population of children born to PRE and non-PRE mothers. In addition, in each of the two groups, the association of anthropometric measures, catecholamine (E, NE) concentrations, and $\mathrm{ABP}$ was examined.

\section{SUBJECTS AND METHODS}

Definitions. Preeclampsia was defined as the development of hypertension and proteinuria ( $>300 \mathrm{mg}$ of urinary protein in $24 \mathrm{~h}$ ) after $20 \mathrm{wk}$ of gestation (16). Hypertension was defined as BP $>140 / 90 \mathrm{~mm} \mathrm{Hg}$ or a rise of $30 / 15 \mathrm{~mm}$ $\mathrm{Hg}$ from the baseline level confirmed by two measurements at least $6 \mathrm{~h}$ apart. Full-term applied to babies born at or after $37 \mathrm{wk}$ of gestation and before $42 \mathrm{wk}$ of gestation and preterm applied to babies born before $37 \mathrm{wk}$ of

Abbreviations: ABP, ambulatory blood pressure; AGA, appropriate for gestational age; BMI, body mass index; E, epinephrine; SGA, small for gestational age; NE, norepinephrine; PSEH, parent-specific expected height 
gestation (calculated from the beginning of the last menstrual cycle). SGA was defined as birth weight or length or ponderal index $>2$ SD scores below the respective mean for the gestational age and sex (17). The ponderal index was calculated as [weight $(\mathrm{g}) /$ length $\left.^{3}(\mathrm{~cm})\right] \times 100$. Appropriate for gestational age (AGA) was defined as birth weight, birth length, and ponderal index $\geq-2$ SD scores and $\leq 2$ SD scores of the respective mean for the gestational age and sex (17). Parent-specific expected height (PSEH) was calculated as determined by Pere et al. (18).

Subjects. All participating children were born at Kuopio University Hospital during the 22-mo period from 1984 to 1986 . The PRE group consisted of every third full-term child and all preterm children who were born to PRE mothers during that period. The extremely preterm children born before the 28th gestational week were excluded. By selecting every third full-term PRE child and all preterm PRE children born at 28-36 wk of gestation, our aim was to increase the relative number of cases with severe maternal preeclampsia. Thus, 84 nonmalformed children with maternal preeclampsia formed the PRE group. Of these 84 PRE children, two were excluded (one due to a metabolic disorder and another due to anorexia nervosa), nine were not reached, and 16 refused to take part in this study. Thus, 57 PRE children (67.9\% of the original study population) participated in this study; however, two of them were unwilling to give a blood sample. The PRE children (25 girls and 32 boys; 31 preterm and 26 full term; 15 SGA and 42 AGA) and their 57 control subjects born to non-PRE mothers were matched for sex, gestational age $( \pm 1 \mathrm{wk})$, and size at birth (SGA versus SGA, AGA versus AGA). The matching for size at birth did not succeed in five PRE-non-PRE pairs; consequently, five PRE children born SGA had a control subject born AGA instead of SGA. None of the participating children were exposed to exogenous corticosteroids prenatally. The study protocol was approved by the Research Ethics Committee of Kuopio University Hospital. Informed written consent was obtained from the child and the parents.

Anthropometric measures and pubertal development. Perinatal data, e.g. birth measures and duration of gestation, had previously been obtained from hospital records. At the age of $12 \mathrm{y}$, height was measured with a calibrated Harpenden stadiometer and recorded to the nearest $0.1 \mathrm{~cm}$, and weight was recorded to the nearest $0.1 \mathrm{~kg}$. A complete physical examination was performed for all children by one of the authors (S.T. or E.R.). Pubertal development was assessed according to the Tanner staging scale $(19,20)$. For the statistical analyses, pubertal stage was defined by breast scores for girls and genital scores for boys (scores 1-5). Pubertal development did not differ significantly between the PRE and non-PRE children at age 12 y $(p=0.375$, the marginal homogeneity test).

ABP monitoring. The ABP monitoring was performed with an oscillometric ABP device, Spacelabs 90207 (Spacelabs, Inc., Redmond, WA). The cuff was chosen from two sizes $(13 \times 24$ and $24 \times 32 \mathrm{~cm})$ to cover two thirds of the arm length between the axilla and fossa cubitalis, and it was placed around the nondominant arm of the child. The subject was instructed to relax the arm during each BP measurement. The device measured ABP in 15-min intervals during the daytime $(0700 \mathrm{~h}$ to $2200 \mathrm{~h})$ and in 30-min intervals during the nighttime $(2200 \mathrm{~h}$ to $0700 \mathrm{~h}$ ). The daytime/nighttime periods were corrected according to the diaries kept by the children. During the monitoring, the children were able to go to school and perform their daily activities. The number of acceptable $\mathrm{ABP}$ readings during the monitoring was $85 \pm 6$ (mean \pm SD) in the PRE and $84 \pm 5$ in the non-PRE children (95\% and $97 \%$ of all readings, respectively).
Casual BP measurements. Casual BP was measured with a standard sphygmomanometer during a home visit by one of the authors (S.T. or E.R.). Korotkoff phase I was used to measure the systolic BP, and Korotkoff phase $\mathrm{V}$ the diastolic BP. BP was measured three times in a seated position after 5 min of rest. The mean values of these three readings were used in the analysis.

Laboratory methods. Blood samples were taken in the morning, between 0900 and $1000 \mathrm{~h}$, after an overnight fast. An i.v. cannula was placed in the antecubital vein for blood sampling. After the child had rested for $1 \mathrm{~h}$ in a recumbent position, blood samples were drawn through the cannula. For the analysis of catecholamines, the blood was collected in ice-chilled tubes and centrifuged as soon as possible at $4^{\circ} \mathrm{C}$. Plasma specimens were immediately frozen and stored at $-70^{\circ} \mathrm{C}$ until analyzed by high-performance liquid chromatography combined with electrochemical detection. The intraassay coefficients of variation (CV) were $7.8 \%$ for $\mathrm{NE}$ and $11.0 \%$ for $\mathrm{E}$, and the interassay CVs were $15.2 \%$ and $11.0 \%$, respectively $(15,21)$.

Statistical analyses. Data were analyzed using the statistical program SPSS for Windows, release 10.0 (SPSS Inc., Chicago, IL). All continuous variables were examined for normality with the Kolmogorov-Smirnov test. The paired and independent samples $t$ tests were used in comparing variables with normal distributions, while the Wilcoxon matched-pairs signed rank test and the Mann-Whitney test were used when the variables distributed nonnormally. Confidence intervals were calculated using a critical value from the $t$ distribution due to nonnormal distributions. ABP values were analyzed by the repeated-measures analysis of variance (ANOVA) adjusting for current weight and height between the PRE and non-PRE children. Either Pearson or Spearman correlation coefficients were computed. The logistic regression analysis was used to analyze the factors associated with high systolic ABP in the PRE and non-PRE children. In the multiple logistic regression analyses, the systolic ABP variable was dichotomized in the PRE and non-PRE groups; as the cutoff point, we used the BP value of the highest tertile of the non-PRE group ( $>118 \mathrm{~mm} \mathrm{Hg})$. Twenty-seven $(47.4 \%)$ PRE children passed this limit. A significance level of $p<0.05$ was used for all analyses.

\section{RESULTS}

Anthropometric characteristics at birth and 12 y of age. The anthropometric characteristics at birth and $12 \mathrm{y}$ of age are presented in Table 1. At $12 \mathrm{y}$ of age, the PRE children were significantly shorter than their control subjects (Table 1). However, this finding was explained by a difference in the paternal heights between the PRE and non-PRE groups (175.3 versus $178.8 \mathrm{~cm} ; p=0.001, n=51$ ). No difference was found in the mean height between the PRE and non-PRE mothers (165.0 versus $164.2 \mathrm{~cm} ; p=0.515, n=57$ ). The mean PSEH did not differ between the PRE and non-PRE groups $(-0.03$ versus 0.09 SD scores; $p=0.269$ ), but adjustment for PSEH eliminated the difference in current height between the PRE and non-PRE groups (0.22 versus $0.45 \mathrm{SD} ; p=0.592)$. The

Table 1. Anthropometric characteristics at birth and 12 y age in PRE and non-PRE children (means [95\% confidence intervals]) are presented

\begin{tabular}{|c|c|c|c|}
\hline Variable & PRE group $(n=57)$ & non-PRE group $(n=57)$ & $p$ value* \\
\hline \multicolumn{4}{|l|}{ At birth } \\
\hline Gestational age (wk) & $36.6(35.7,37.5)$ & $36.6(35.7,37.5)$ & 0.480 \\
\hline Weight $(\mathrm{g})$ & $2592(2385,2799)$ & $2865(2646,3084)$ & $<0.001$ \\
\hline Length $(\mathrm{cm})$ & $46.7(45.6,47.8)$ & $47.5(46.4,48.6)$ & 0.036 \\
\hline Length (SD scores) & $-0.71(-1.06,-0.36)$ & $-0.31(-0.65,0.03)$ & 0.070 \\
\hline Ponderal index $\left(\mathrm{g} / \mathrm{cm}^{3}\right)$ & $2.45(2.38,2.52)$ & $2.59(2.51,2.67)$ & 0.001 \\
\hline Weight $(\mathrm{kg})$ & $43.8(41.5,46.1)$ & $46.3(43.3,49.3)$ & 0.289 \\
\hline Weight for height (\%) & $6(2-10)$ & $6(1-11)$ & 0.704 \\
\hline Height $(\mathrm{cm})$ & $152.0(150.0,154.0)$ & $155.0(153.0,157.0)$ & 0.018 \\
\hline Height (SD scores) & $0.16(-0.13,0.45)$ & $0.56(0.30,0.82)$ & 0.016 \\
\hline BMI $\left(\mathrm{kg} / \mathrm{m}^{2}\right)$ & $18.8(18.0,19.6)$ & $19.1(18.1,20.1)$ & 0.581 \\
\hline
\end{tabular}

* Wilcoxon matched pairs signed rank test for differences. 
mean current weight and BMI did not differ between the PRE and non-PRE children (Table 1).

ABP in the PRE and non-PRE children. The PRE children had significantly higher mean 24-h systolic and diastolic ABPs than the non-PRE children. The same was true for the mean daytime and nighttime systolic and diastolic ABPs in the current height- and weight-adjusted analyses (Fig. 1). The daytime systolic ABP was higher than the nighttime systolic ABP in both the PRE (122.0 versus $108.3, p<0.001)$ and in the non-PRE children $(117.8$ versus $105.9, p<0.001)$. Similarly, the daytime diastolic ABP was higher than the nighttime diastolic ABP in both groups (73.5 versus 59.1, $p<$ $0.001 ; 69.7$ versus $56.6, p<0.001$, respectively). The percentages for the nocturnal decrease of systolic and diastolic $\mathrm{ABP}$ values were not different in the PRE and non-PRE children (the decrease for the systolic means $11.2 \%$ versus $10.0 \%, p=0.150$; and for the diastolic means $19.4 \%$ versus $18.4 \%, p=0.443$, respectively).

In subgroup analysis, the mean 24-h systolic and diastolic ABPs did not differ significantly between the PRE children born SGA and AGA, nor did the 24-h systolic and diastolic ABPs differ between the preterm and full-term PRE children (data not shown). However, the PRE boys $(n=32)$ had significantly higher 24-h systolic ABP than the PRE girls $(n=$ 25) (120.3 versus $117.5, p=0.047)$.

Comparison between casual BP and 24-h ABP values. The mean casual systolic BP values were significantly lower than the mean 24-h systolic ABP values in both the PRE and non-PRE group (116.8 versus $119.1, p=0.014 ; 113.0$ versus 115.3; $p=0.010$, respectively). On the contrary, the mean casual diastolic BP values were significantly higher than the mean 24-h diastolic ABP values in the PRE and non-PRE groups (74.3 versus $70.3, p<0.001 ; 70.5$ versus $67.0, p=$ 0.001, respectively). The mean 24-h systolic ABP correlated positively with the mean casual systolic BP in the PRE and non-PRE children $(r=0.674, p<0.001 ; r=0.695, p<$ 0.001 , respectively). Similarly the mean 24 -h diastolic ABP correlated positively with the mean casual diastolic BP in the

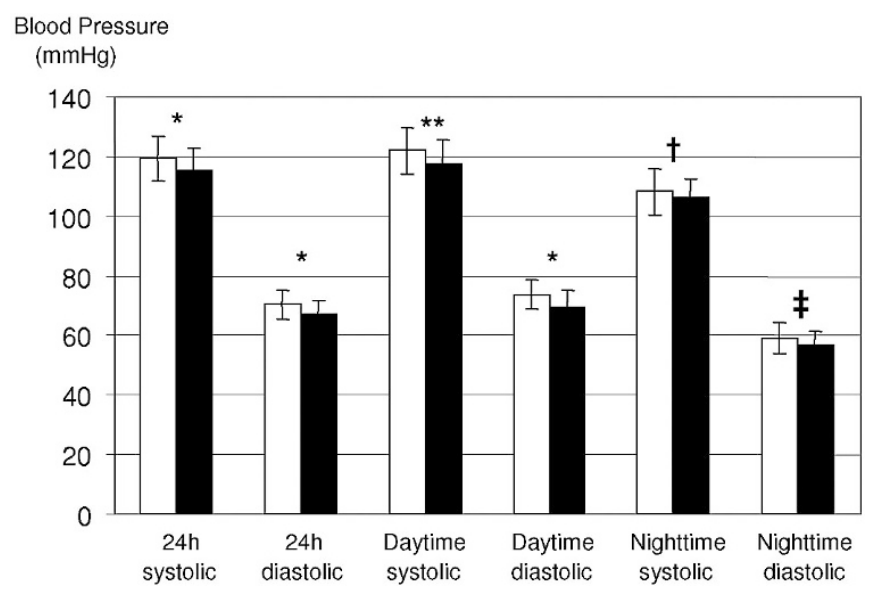

Figure 1. The means ( \pm SD) of 24-h, daytime, and nighttime systolic and diastolic ABP values in the PRE (open columns) and non-PRE (filled columns) children. Comparisons by repeated-measures ANOVA adjusted by current height and weight. ${ }^{*} p=0.001 ; * * p<0.001 ; \dagger p=0.020 ; \ddagger p=0.015$.
PRE and non-PRE children $(r=0.591, p<0.001 ; r=0.402$, $p=0.002$, respectively).

Plasma E and NE concentrations. In the PRE children, the mean plasma $\mathrm{E}$ concentration tended to be higher than in the non-PRE children $(0.32$ versus $0.27 \mathrm{nmol} / \mathrm{L}, p=0.087)$, whereas the plasma NE concentrations were similar in the groups ( 1.40 versus. $1.44 \mathrm{nmol} / \mathrm{L}, p=0.680$, respectively). The mean plasma E levels tended to be higher in the SGA children in both PRE and non-PRE groups ( 0.36 and 0.37 $\mathrm{nmol} / \mathrm{L}$, respectively) when compared with the AGA children in the PRE and non-PRE groups $(0.30$ and $0.25 \mathrm{nmol} / \mathrm{L}$, respectively). However, the differences between the SGA and AGA subjects were not significant.

Correlations between ABP, anthropometric characteristics, and plasma $E$ and $N E$ concentrations. The $\mathrm{ABP}$ variables did not correlate with the anthropometric measures at birth or at age $12 \mathrm{y}$ in the PRE children. The 24-h systolic ABP correlated positively with the current height (expressed in SD scores), current weight for height, and BMI in the non-PRE children $(r=0.316, p=0.017 ; r=0.468, p<$ $0.001 ; r=0.472, p<0.001$, respectively). The 24-h diastolic ABP correlated also positively with the current weight for height and BMI in the non-PRE children $(\mathrm{r}=0.313, p=$ $0.018 ; r=0.275, p=0.039$, respectively).

The plasma E concentrations correlated positively with the 24-h systolic and diastolic ABPs in the PRE children $(r=$ $0.294, p=0.029 ; r=0.334, p=0.013$, respectively), but not in the non-PRE children. Plasma NE did not correlate with the $\mathrm{ABP}$ variables in the PRE and non-PRE children.

Factors associated with systolic ABP. According to the puberty-adjusted multiple logistic regression analysis, high plasma E concentration and being born SGA were associated with high 24-h systolic ABP levels in the PRE children. An increase of $0.10 \mathrm{nmol} / \mathrm{L}$ in plasma $\mathrm{E}$ concentration increased by 2.3 -fold the risk of high 24-h systolic ABP. The PRE children who were born SGA had an 8.7-fold risk of elevated 24-h systolic ABP (Table 2). In the non-PRE group, high BMI and plasma E level predicted high 24-h systolic ABP (Table $3)$.

\section{DISCUSSION}

This cohort study with matched pairs revealed that the PRE children had significantly higher systolic and diastolic ABPs than the non-PRE children. In logistic regression analysis, high plasma E concentration and being born SGA were asso-

Table 2. Factors associated with high 24-h systolic ABP in the PRE children: results of multiple logistic regression analysis $(n=55)$

\begin{tabular}{lcccc}
\hline Variables* & $\begin{array}{c}\text { Regression } \\
\text { coefficient }\end{array}$ & Significance & OR & $\begin{array}{l}95 \% \text { CI } \\
\text { for OR }\end{array}$ \\
\hline Male sex & 1.70 & 0.065 & 5.4 & $0.9-33$ \\
SGA & 2.2 & 0.024 & 8.7 & $1.3-57$ \\
High BMI $\left(\mathrm{kg} / \mathrm{m}^{2}\right.$ ) & 0.24 & 0.094 & 1.3 & $1.0-1.7$ \\
Plasma E (unit $0.10 \mathrm{nmol} / \mathrm{L})$ & 0.83 & 0.008 & 2.3 & $1.2-4.3$ \\
\hline
\end{tabular}

Variation explained by the model: $44.5 \%$. OR, odds ratio; CI, confidence interval.

* Adjusted for pubertal stage. 
Table 3. Factors associated with high 24-h systolic ABP in the non-PRE children: results of multiple logistic regression analysis $(n=55)$

\begin{tabular}{lcccl}
\hline Variables* & $\begin{array}{c}\text { Regression } \\
\text { coefficient }\end{array}$ & Significance & OR & $\begin{array}{l}95 \% \mathrm{CI} \\
\text { for OR }\end{array}$ \\
\hline Male sex & 0.59 & 0.448 & 1.8 & $0.4-8.4$ \\
$\mathrm{SGA}$ & 0.76 & 0.436 & 2.1 & $0.3-14$ \\
High BMI $\left(\mathrm{kg} / \mathrm{m}^{2}\right)$ & 0.29 & 0.005 & 1.3 & $1.1-1.6$ \\
Plasma E (unit $0.10 \mathrm{nmol} / \mathrm{L})$ & 0.63 & 0.039 & 1.9 & $1.0-3.4$ \\
\hline
\end{tabular}

Variation explained by the model: $36.4 \%$.

* Adjusted for pubertal stage.

ciated with high 24-h systolic ABP in the PRE children. In a similar analysis in the non-PRE children, current BMI and high plasma E level predicted high 24-h systolic ABP.

According to previous reports, maternal preeclampsia seems to be associated with elevated BP in an offspring even during childhood and adolescence (13-15). Palti and Rothschild (13) reported that 6-y-old boys (but not girls) born to PRE mothers had higher diastolic BPs than their control subjects born to non-PRE mothers. In that study, the systolic BP values did not differ between the cases and controls. Seidman et al. (14) found that 17-y-old girls with maternal preeclampsia had higher systolic and diastolic BPs than their control subjects, whereas only the mean systolic BP differed between the male subjects at the same age. We reported previously the results of casual BP measurements in a study population almost identical to the present one (15). The PRE children $(n=60)$ in that analysis had significantly higher casual systolic and diastolic BPs than their matched control subjects. Our present results of the ABP monitoring show that PRE children have systolic and diastolic BPs higher than those of their controls throughout the day. Furthermore, our findings suggest that maternal preeclampsia may have a greater influence on BP level in male than in female subjects at $12 \mathrm{y}$ of age.

ABP monitoring in children is a useful tool in distinguishing constant BP elevation from "white coat hypertension," and the validity of the Spacelabs monitor we used has been confirmed previously (22). Soergel et al. (23) studied ABP values of oscillometric 24-h monitoring in a large population of healthy children and adolescents to produce normal ABP values for the aforementioned age groups. In the present study, the systolic ABP values were about $8 \%$ higher in the PRE children and 5\% higher in the non-PRE children than in the subjects with similar heights in the study of Soergel et al. (23). The nocturnal fall or "dipping" of BP is a physiologic phenomenon, and its absence has been considered a predictor of cardiovascular morbidity and mortality (24). However, in our study population, the percentages of nocturnal ABP dipping were quite close to those reported by Soergel et al. (23), and the decrease in nocturnal systolic and diastolic ABP did not differ between the PRE and non-PRE children. Accordingly, the normal diurnal BP variation was observed in both the PRE and non-PRE children.

The role of the sympathetic system in the pathophysiology of preeclampsia is obscure. In previous studies, plasma catecholamine concentrations have been found to be increased in PRE pregnant women $(4,25,26)$, and increased catecholamine secretion was supposed to be of placental origin (26). In our study, the PRE children tended to have slightly higher circulating E levels than the non-PRE children. This finding needs to be confirmed in independent studies. The methodology for plasma catecholamine measurements is demanding, as shown by the relatively high $\mathrm{CV}$ in the $\mathrm{E}$ and $\mathrm{NE}$ assays. The highest concentrations of plasma $\mathrm{E}$ were found in PRE and non-PRE children born SGA. This finding is in accordance with a previous separate study showing that 12 -y-old children born SGA at term had higher plasma E levels than their control subjects born AGA (27).

The relationship between low birth weight and elevated BP in children and adults has been shown in many studies $(28,29)$. Preeclampsia leads in about $30 \%$ of cases to fetal growth restriction (30). Accordingly, being born SGA could be an additional risk factor for elevated BP in children born after a PRE pregnancy. It is presumable that in pregnancies terminating preterm, preeclampsia is often severe and leads to fetal growth restriction. In the present study, being born SGA predicted high 24-h systolic ABP in the PRE children. However, premature birth did not influence any $A B P$ variables.

In summary, systolic and diastolic $\mathrm{BP}$ values in $\mathrm{ABP}$ monitoring were elevated in the PRE children. High plasma E concentration and being born SGA are associated with high systolic 24-h ABP in the PRE children. Presumably maternal preeclampsia affects offspring via several mechanisms, including genetic ones and metabolic consequences of restricted intrauterine growth.

\section{REFERENCES}

1. Cooper DW, Brennecke SP, Wilton AN 1993 Genetics of pre-eclampsia. Hypertens Pregnancy 12:1-23

2. Roberts JM, Cooper DW 2001 Pathogenesis and genetics of pre-eclampsia. Lancet 357:53-56

3. Roberts JM, Redman CW 1993 Pre-eclampsia: more than pregnancy-induced hypertension. Lancet 341:1447-1451

4. Kaaja RJ, Moore MP, Yandle TG, Ylikorkala O, Frampton CM, Nicholls MG 1999 Blood pressure and vasoactive hormones in mild preeclampsia and normal pregnancy. Hypertens Pregnancy 18:173-187

5. Agatisa PK, Ness RB, Roberts JM, Constantino JP, Kuller LH, McLaughlin MK 2004 Impairment of endothelial function in women with a history of preeclampsia: an indicator of cardiovascular risk. Am J Physiol Heart Circ Physiol 286:H1389$\mathrm{H} 1393$

6. Jonsdottir LS, Arngrimsson R, Geirsson RT, Sigvaldason H, Sigfusson N 1995 Death rates from ischaemic heart disease in women with a history of hypertension in pregnancy. Acta Obstet Gynecol Scand 74:772-776

7. Hannaford P, Ferry S, Hirsch S 1997 Cardiovascular sequelae of toxaemia of pregnancy. Heart 77:154-158

8. Irgens HU, Reisæter L, Irgens LM, Lie RT 2001 Long-term mortality of mothers and fathers after pre-eclampsia: population based cohort study. BMJ 323:1213-1217

9. Haukkamaa L, Salminen M, Laivuori H, Leinonen H, Hiilesmaa V, Kaaja R 2004 Risk for subsequent coronary artery disease after preeclampsia. Am J Cardiol 93:805-808

10. Laivuori H, Tikkanen MJ, Ylikorkala O 1996 Hyperinsulinemia 17 years after preeclamptic first pregnancy. J Clin Endocrinol Metab 81:2908-2911

11. Kaaja R, Laivuori H, Laakso M, Tikkanen MJ, Ylikorkala O 1999 Evidence of a state of increased insulin resistance in preeclampsia. Metabolism 48:892-896

12. Barden AE, Beilin LJ, Ritchie J, Walters BN, Michael C 1999 Does a predisposition to the metabolic syndrome sensitize women to develop pre-eclampsia? J Hypertens 17:1307-1315

13. Palti H, Rothschild E 1989 Blood pressure and growth at 6 years of age among offsprings of mothers with hypertension of pregnancy. Early Hum Dev 19:263-269

14. Seidman DS, Laor A, Gale R, Stevenson DK, Mashiach S, Danon YL 1991 Pre-eclampsia and offspring's blood pressure, cognitive ability and physical development at 17-years-of-age. Br J Obstet Gynaecol 98:1009-1014

15. Tenhola S, Rahiala E, Martikainen A, Halonen P, Voutilainen R 2003 Blood pressure, serum lipids, fasting insulin, and adrenal hormones in 12-year-old children born with maternal preeclampsia. J Clin Endocrinol Metab 88:1217-1222

16. Davey DA, MacGillivray I 1988 The classification and definition of the hypertensive disorders of pregnancy. Am J Obstet Gynecol 158:892-898 
17. Pihkala J, Hakala T, Voutilainen P, Raivio K 1989 New Finnish fetal growth charts (in Finnish). Duodecim 105:1540-1546

18. Pere A, Perheentupa J, Peter M, Voutilainen R 1995 Follow up of growth and steroids in premature adrenarche. Eur J Pediatr 154:346-352

19. Marshall WA, Tanner JM 1969 Variations in pattern of pubertal changes in girls. Arch Dis Child 44:291-303

20. Marshall WA, Tanner JM 1970 Variations in pattern of pubertal changes in boys. Arch Dis Child 45:13-16

21. Nyyssönen K, Parviainen MT 1987 Practical observations and sources of error in assays of plasma catecholamines by "high-performance" liquid chromatography with electrochemical detection. Clin Chem 33:1938-1939

22. O'Brien E, Mee F, Atkins N, O'Malley K 1991 Accuracy of the Space-Labs 90207 determined by the British Hypertension Society Protocol. J Hypertens 9(suppl 5):S25-S31

23. Soergel M, Kirschstein M, Busch C, Danne T, Gellerman J, Holl R, Krull F, Reichert H, Reusz GS, Rascher W 1997 Oscillometric twenty-four-hour ambulatory blood pressure values in healthy children and adolescents: a multicenter trial including 1141 subjects. J Pediatr 130:178-184
24. Gibbs CR, Murray S, Beevers DG 1998 The clinical value of ambulatory blood pressure monitoring. Heart. 79:115-117

25. Øian P, Kjeldsen SE, Eide I, Maltau JM 1986 Increased arterial catecholamines in pre-eclampsia. Acta Obstet Gynecol Scand 65:613-617

26. Manyonda IT, Slater DM, Fenske C, Hole D, Choy MY, Wilson C 1998 A role for noradrenaline in pre-eclampsia: towards a unifying hypothesis for the pathophysiology. Br J Obstet Gynaecol 105:641-648

27. Tenhola S, Martikainen A, Rahiala E, Parviainen M, Halonen P, Voutilainen R 2002 Increased adrenocortical and adrenomedullary hormonal activity in 12-year-old children born small for gestational age. J Pediatr 141:477-482

28. Law CM, Shiell AW 1996 Is blood pressure inversely related to birth weight? The strength of evidence from a systematic review of the literature. J Hypertens 14:935-941

29. Rahiala E, Tenhola S, Vanninen E, Herrgård E, Tikanoja T, Martikainen A 2002 Ambulatory blood pressure in 12-year-old children born small for gestational age. Hypertension 39:909-913

30. Walker JJ 2000 Pre-eclampsia. Lancet 356:1260-1265 